\title{
Rasmussen's encephalitis with persistent epilepsy in a young man
}

\author{
Van Trung Hoang ${ }^{1}$ and Vichit Chansomphou ${ }^{2}$ \\ ${ }^{1}$ Thien Hanh Hospital \\ ${ }^{2}$ Savannakhet Medical-Diagnostic Center
}

September 11, 2020

\begin{abstract}
Rasmussen's encephalitis is a rare chronic neurological disorder, characterized by unilateral inflammation of the cerebral cortex, drug-resistant epilepsy, progressive neurological and cognitive impairment. We report a case of a 17-year old male with persistent epilepsy, intellectual impairment, and cognitive deterioration.
\end{abstract}

\section{KEYWORDS}

Chronic neurological disorder, Hemimegalencephaly, Persistent seizures, Rasmussen's encephalitis

\section{KEY CLINICAL MESSAGE}

Rasmussen's encephalitis (RE) is an uncommon cause of the seizure. Important key findings of RE include intractable seizure activity in children, progressive atrophy of the involved hemisphere, small hemisphere with the large ventricle.

\section{CASE DESCRIPTION}

A 17-year-old male was admitted to the hospital for persistent seizures. Symptoms began five years ago. They lasted an average of one minute and would stop and restart, eventually occurring up to multiple times. Consciousness was preserved with the events. Physical examination revealed intellectual and cognitive impairment. Computed tomography image showed atrophy of the left cerebral hemisphere (Figure 1). Clinical and imaging features were consistent with the diagnosis of RE. This patient had been attempted treatment with conventional therapies including steroids, plasmapheresis, and intravenous immunoglobulin. The condition had improved, but not completely.

$\mathrm{RE}$ is a rare chronic neurological disorder, characterized by unilateral inflammation of the cerebral cortex, drug-resistant epilepsy, progressive neurological and cognitive impairment. The etiology is unknown, but evidence supports an autoimmune basis. ${ }^{1}$ Diagnostic imaging methods, especially magnetic resonance imaging, have become a mainstay for diagnostic assessment and follow-up in RE. Usually, in the acute stage, there is a hyperintense signal on the T2-weighted image that is often present in cortical/subcortical regions or both. The distribution is heterogeneous and temporal fluctuation. Thereafter, most patients present with unilateral enlargement of the ventricular system. RE requires a differential diagnosis from hemimegalencephaly. Treatment with immunotherapy can improve at an early stage, at a later stage, hemispherectomy should be considered. $^{2}$

\section{CONFLICT OF INTEREST}

The authors declare no conflicts of interest.

\section{AUTHOR CONTRIBUTIONS}


VTH: involved in patient management and wrote the manuscript. VTH and VC: contributed to the critical review of the manuscript.

\section{INFORMED CONSENT}

Informed consent was obtained from the patients prior to conducting this study.

\section{REFERENCES}

1. Varadkar S, Bien CG, Kruse CA, Jensen FE, Bauer J, Pardo CA, et al. Rasmussen's encephalitis: clinical features, pathobiology, and treatment advances. Lancet Neurol . 2014;13(2):195-205.

2. Bien CG, Widman G, Urbach H, Sassen R, Kuczaty S, Wiestler OD, et al. The natural history of Rasmussen's encephalitis. Brain . 2002;125(Pt 8):1751-1759.

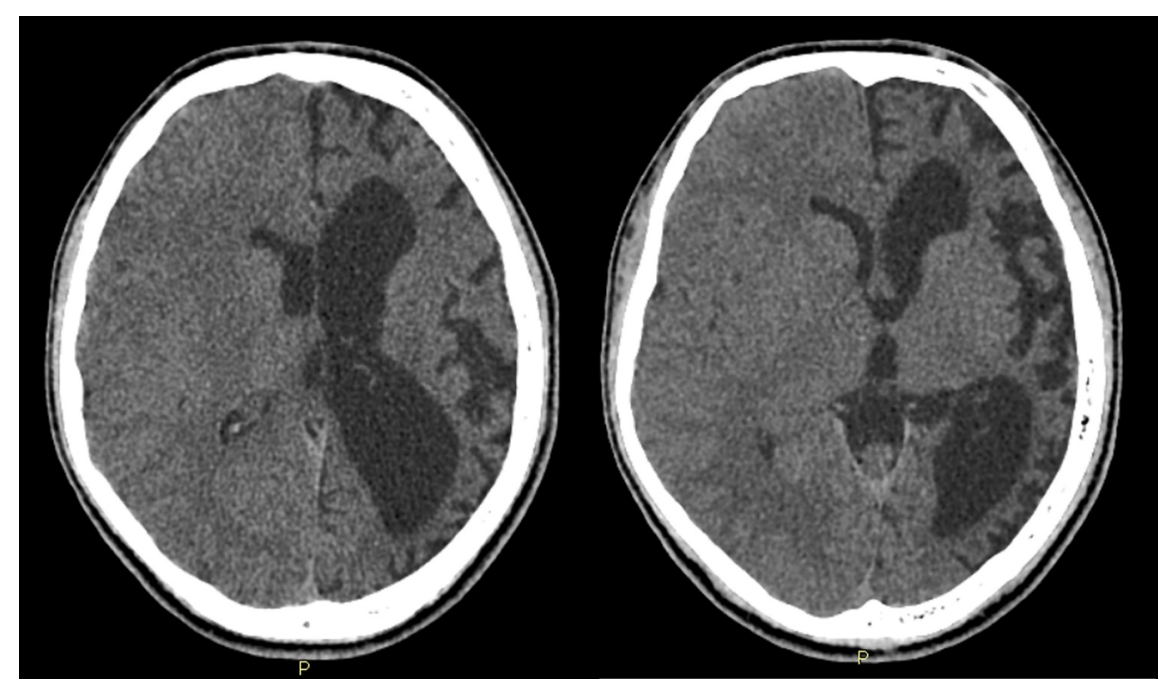

FIGURE 1 Non-contrast computed tomography of the brain shows atrophy of the left cerebral hemisphere with dilatation of the left lateral ventricle. 